\title{
Evaluation of XRD analysis of amorphous alloys
}

\author{
G. Körösy ${ }^{1, a^{*}}$, K. Tomolya ${ }^{1, b}$ D. Janovszky ${ }^{1, c}$ J. Sólyom ${ }^{1, d}$ \\ ${ }^{1}$ MTA-ME Materials Science Research Group, Miskolc-Egyetemvaros, H-3515, Hungary \\ a*valaki121@gmail.com, bfemkinga@uni-miskolc.hu, cfekjd@uni-miskolc.hu, dfemsj@uni- \\ miskolc.hu
}

Keywords: amorphous alloy, casting, milling, X-ray diffraction

\begin{abstract}
Amorphous/nanocrystalline alloys were prepared by centrifugal casting and mechanical milling and analysed the amorphous fraction. Amorphous alloys have a typical XRD pattern, because in these systems the degree of regularity of atomic positions is very small. Liquids and glasses generate only one ore more broad diffuse halos. In order to calculate the amorphous fraction value, the different between the crystalline and the amorphous part of the patterns need to be realize. Furthermore, the measured values need to be smoothed before the determination of the degree of the amorphous fraction. A software developed by us, called GerKiDo can separate and calculate the amorphous fraction from the XRD pattern. These calculated values are compared to the results calculated by the software settled in the X-ray equipment and amorphous fraction measured by DSC. The evaluation of X-Ray diffractograms is often time-consuming and difficult. The motivation of this paper was to develop a cost effective, fast and useable method to define the amorphous fraction of samples produced by different technologies.
\end{abstract}

